\title{
$\mathcal{P}$-kernel normal systems for $\mathcal{P}$-inversive semigroups
}

\section{Hengwu Zheng}

Received: 20 January 2010 / Accepted: 29 June 2010 / Published online: 9 July 2011

(C) The Author(s) 2011. This article is published with open access at Springerlink.com

\begin{abstract}
As a generalization of Preston's kernel normal systems, $\mathcal{P}$-kernel normal systems for $\mathcal{P}$-inversive semigroups are introduced, and strongly regular $\mathcal{P}$-congruences on $\mathcal{P}$-inversive semigroups in terms of their $\mathcal{P}$-kernel normal systems are characterized. These results generalize the corresponding results for $\mathcal{P}$-regular semigroups and $\mathcal{P}$-inversive semigroups.
\end{abstract}

Keywords E-inversive semigroup $\cdot \mathcal{P}$-inversive semigroup $\cdot$ Strongly regular $\mathcal{P}$-congruence $\cdot \mathcal{P}$-kernel normal system

\section{Introduction}

For standard terminology and notation in semigroup theory see Howie [11]. As usual, $E(S)$ is the set of idempotents of a semigroup $S, V(a)=\left\{a^{\prime} \in S: a a^{\prime} a=a, a^{\prime} a a^{\prime}=\right.$ $\left.a^{\prime}\right\}$ is the set of all inverses of $a \in S$.

A semigroup $S$ is $E$-inversive if for every $a \in S$ there exists $x \in S$ such that $a x$ is idempotent. This concept was introduced by Thierrin [25]. A semigroup is an $E$ semigroup if its idempotents form a subsemigroup. Basic properties of E-inversive semigroups were given by Blyth and Almeida Santos [1], Catino and Miccoli [2], Hayes [9, 10], Mitsch and Petrich [18] and Mitsch [15-17]. From Lemma 3.1 in [15], a semigroup $S$ is E-inversive if and only if

$$
(\forall a \in S) \quad W(a)=\left\{a^{\prime} \in S: a^{\prime}=a^{\prime} a a^{\prime}\right\} \neq \emptyset .
$$

The elements of $W(a)$ are called weak inverses of $a$.

Communicated by Thomas E. Hall.

H. Zheng $(\bowtie)$

School of Mathematical Sciences, Qufu Normal University, Qufu, Shandong 273165, P.R. China

e-mail: hwzheng163@163.com 
Preston [21] introduced kernel normal systems to characterize congruences on inverse semigroups. Meakin [14] gave a generalization of kernel normal systems for orthodox semigroups. Imaoka [12] obtained a generalization of Preston's kernel normal systems for regular *-semigroups. A semigroup $S$ is a regular *-semigroup if $S$ with a unary operation * satisfies $\left(a^{*}\right)^{*}=a,(a b)^{*}=b^{*} a^{*}$ and $a a^{*} a=a$ for any $a, b \in S$ (see $[12,19])$. We have seen that orthodox semigroups and regular *-semigroups are within the class of $\mathcal{P}$-regular semigroups. A $\mathcal{P}$-regular semigroup $S(P)$ is a regular semigroup $S$ whose set of idempotents $E(S)$ includes a subset $P$ with properties $P^{2} \subseteq E(S), p P p \subseteq P$ for all $p \in P$, and $\left\{a^{\prime} \in V(a): a P^{1} a^{\prime} \cup a^{\prime} P^{1} a \subseteq P\right\} \neq \emptyset$ for all $a \in S$, where $P^{1}=P \cup\{1\}$ (see [28, 29]). Sen [23] gave a characterization of $\mathcal{P}$ congruences (the terminology a $\mathcal{P}$-congruence for a usual congruence on a $\mathcal{P}$-regular semigroup [28]) on $\mathcal{P}$-regular semigroups in terms of their $\mathcal{P}$-kernel normal systems.

The existence of the least group congruence on an E-inversive semigroup was noted by Hall and Munn [8]. An explicit description of this congruence was given by Mitsch [15]. The least group congruence on an E-inversive E-semigroup was characterized by Reither [22]. Further characterizations of group congruences on an E-inversive semigroup has been obtained by the author [30]. An alternative description of this congruence on an E-inversive E-semigroup was given by Weipoltshammer [27]. Blyth and Almeida Santos [1] gave an alternative description of group congruences on an E-inversive semigroup.

Weipoltshammer [27] described some congruences on E-inversive E-semigroups. References [4, 5] and [3] refer to work by Fan and Chen in which $\mathcal{P}$-inversive semigroups where first studied (using the so called $\mathcal{P}$-kernel normal systems) and the regular $\mathcal{P}$-congruences on $\mathcal{P}$-inversive semigroups. A $\mathcal{P}$-inversive semigroup is an E-inversive semigroup whose set of idempotents includes a subset $P$ with properties that make it suitable in a "kernel normal systems" that generalizes the systems of Preston [21] and Meakin [14] in their respective congruence theories for inverse and orthodox semigroups.

Definition 1.1 (see [3-5]) An E-inversive semigroup $S$ is called a $\mathcal{P}$-inversive semigroup, if there exists a nonempty subset $P$ of $E(S)$ such that

(1) $P^{2} \subseteq E(S)$;

(2) $(\forall p \in P) p P p \subseteq P$;

(3) $(\forall a \in S) W_{P}(a)=\left\{a^{\prime} \in W(a): a P^{1} a^{\prime} \cup a^{\prime} P^{1} a \subseteq P\right\} \neq \emptyset$, where $P^{1}=P \cup\{1\}$.

The subset $P$ of $E(S)$ satisfying (1)-(3) above is called a characteristic set ( $C$-set, for short) of $S$, each element in $W_{P}(a)$ is called a weak $\mathcal{P}$-inverse of $a$. Clearly, for any $p \in P$, we have $p \in W_{P}(p)$. Because of the central role of $P$ in $S$ we denote a $\mathcal{P}$-inversive semigroup $S$ with the $C$-set $P$ by $S(P)$. Throughout this paper, $S(P)$ is always an arbitrary $\mathcal{P}$-inversive semigroup.

From Definition 1.1 it follows that all $\mathcal{P}$-regular semigroups and E-inversive E-semigroups are within the class of $\mathcal{P}$-inversive semigroups. We have seen that there exist $\mathcal{P}$-inversive semigroups which are neither $\mathcal{P}$-regular nor E-inversive $\mathrm{E}$ semigroups (see [4] and [7]).

A regular $\mathcal{P}$-congruence $\rho$ on $S(P)$ is a congruence $\rho$ with property a $\rho a a^{\prime} a$ for all $a \in S(P)$ and all $a^{\prime} \in W_{P}(a)$ in the sense of [3]. 
In [7], the strong $\mathcal{P}$-congruences and some sublattices of the strong $\mathcal{P}$-congruence lattice on a $\mathcal{P}$-inversive semigroup were studied. A strong $\mathcal{P}$-congruence $\rho$ on $S(P)$ is a congruence $\rho$ with properties for all $a, b \in S(P), a \rho b$ implies $a^{\prime} \rho b^{\prime}$ for all $a^{\prime} \in W_{P}(a), b^{\prime} \in W_{P}(b)$, and $a \rho a a^{\prime} a$ for all $a \in S(P)$ and all $a^{\prime} \in W_{P}(a)$ in the sense of [4]. Siripitukdet and Sattayaporn [24] characterized the maximum idempotent separating congruence on an E-inversive E-semigroup.

The article [13] by Lou, Fan and Li is a major comprehensive paper on this topic. In it, any regular congruence on an arbitrary E-inversive semigroup is uniquely specified in terms of its trace and kernel (generalizing results for regular semigroups by Feigenbaum [6], Trotter [26] and especially by Pastijn and Petrich [20]). In the final section of [13], the regular congruences on E-inversive semigroups are characterized in terms of kernel normal systems.

The purpose of this paper is to generalize Preston's kernel normal systems to $\mathcal{P}$ inversive semigroups, and to give a description of strongly regular $\mathcal{P}$-congruences on $\mathcal{P}$-inversive semigroups in terms of their $\mathcal{P}$-kernel normal systems. The type of technique used here is basically the one used in [23]. These results generalize the corresponding results for $\mathcal{P}$-regular semigroups [23] and $\mathcal{P}$-inversive semigroups [3, 5].

By using the weak inverses in semigroups, Lou etc. [13] described regular congruences on E-inversive semigroups in terms of their kernel normal systems. In this paper we show that weak $\mathcal{P}$-inverses indeed can replace weak inverses in a congruence theory for $\mathcal{P}$-inversive semigroups, in a neater manner than that obtained in [13].

Recall that a congruence $\rho$ on a semigroup $S$ is said to be regular if $S / \rho$ is a regular semigroup. Obviously, a congruence satisfying the property

$$
(\forall a \in S)\left(\exists a^{\prime} \in W_{P}(a)\right) \quad a \rho a a^{\prime} a
$$

on $S(P)$ is regular. In general, regular congruences on $S(P)$ may not satisfy the property (P). For example, let $S=(\mathbb{N}, \cdot)$ be the multiplicative semigroup of all nonnegative integers and $\rho$ be the congruence determined by the partition:

$$
\{0\},\{m \in \mathbb{N}: m>0\} .
$$

Then $S$ is an E-inversive semigroup with $E(S)=\{0,1\}$, and $S / \rho=\{\overline{0}, \overline{1}\}$ is a band. Let $P=\{0\}$. Certainly, $S(P)$ is $\mathcal{P}$-inversive, and $\rho$ is regular. But there is no weak $\mathcal{P}$-inverse $x$ of 1 such that $1 \rho 1 x 1$. In this paper we shall be interested in the regular congruences which possesses the property $(\mathrm{P})$ and we call such congruences strongly regular. That is,

Definition 1.2 A congruence $\rho$ on $S(P)$ is called a strongly regular $\mathcal{P}$-congruence, if it satisfies

$$
(\forall a \in S)\left(\exists a^{\prime} \in W_{P}(a)\right) \quad a \rho a a^{\prime} a .
$$


Example 1.3 Let $S$ be the semigroup with Cayley table [27, Example 5.1]):

\begin{tabular}{c|lllllll}
$S$ & $a$ & $b$ & $c$ & $d$ & $e$ & $f$ & $g$ \\
\hline$a$ & $e$ & $g$ & $a$ & $e$ & $e$ & $g$ & $e$ \\
$b$ & $c$ & $d$ & $e$ & $d$ & $e$ & $e$ & $f$ \\
$c$ & $e$ & $f$ & $c$ & $e$ & $e$ & $f$ & $e$ \\
$d$ & $e$ & $d$ & $e$ & $d$ & $e$ & $e$ & $e$ \\
$e$ & $e$ & $e$ & $e$ & $e$ & $e$ & $e$ & $e$ \\
$f$ & $c$ & $e$ & $e$ & $e$ & $e$ & $e$ & $f$ \\
$g$ & $a$ & $e$ & $e$ & $e$ & $e$ & $e$ & $g$
\end{tabular}

Then $S$ is an E-inversive E-semigroup with $E(S)=\{c, d, e, g\}$. Let $P=E(S)$. Then $S(P)$ is $\mathcal{P}$-inversive. Let $\rho$ be the congruence determined by the partition $\{a\},\{b, f\},\{c\},\{d, e\},\{g\}$. Notice that $b$ is the only non-regular element of $S, a \in$ $W_{P}(b)$, and $b a b=f \rho b$, we have that $\rho$ is a strongly regular $\mathcal{P}$-congruence on $S(P)$. Now $d \in W_{P}(b)$ and $b d b=d$, but $d$ and $b$ are not $\rho$-related. This implies that $\rho$ is not a regular $\mathcal{P}$-congruence on $S(P)$. Certainly, $\rho$ is not a strong $\mathcal{P}$-congruence on $S(P)$.

The previous example illustrates that the concept of strong regular $\mathcal{P}$-congruences on $\mathcal{P}$-inversive semigroups is a generalization of the concept of strong $\mathcal{P}$-congruences and regular $\mathcal{P}$-congruences on this class of semigroups.

\section{$2 \mathcal{P}$-kernel normal systems}

The set $\mathcal{B}=\left\{B_{i}: i \in I\right\}$ of subsemigroups of $S(P)$ is said to be a $\mathcal{P}$-kernel normal system for $S(P)$ if the following conditions hold:

(K1) $B_{i} \cap B_{j}=\emptyset$ if $i \neq j \in I$.

(K2) Each $B_{i}$ contains an element of $P$ and each element of $P$ is contained in some $B_{i}$.

(K3) For any $a \in S(P)$ there exists $a^{\prime} \in W_{P}(a)$ such that

$$
\left(\forall x, y \in S^{1}\right)(\forall B \in \mathcal{B}) x a y \in B \Leftrightarrow x a a^{\prime} a y \in B .
$$

(K4)

$$
\begin{aligned}
& \left(\forall x, y \in S^{1}\right)\left(\forall i_{1}, i_{2}, \ldots, i_{n}, j \in I\right) \\
& \quad x B_{i_{1}} B_{i_{2}} \cdots B_{i_{n}} y \cap B_{j} \neq \emptyset \Rightarrow x B_{i_{1}} B_{i_{2}} \cdots B_{i_{n}} y \subseteq B_{j} .
\end{aligned}
$$

The weak $\mathcal{P}$-inverse $a^{\prime}$ of $a$ satisfying (K3) above is called a weak $\mathcal{B}$-inverse of $a$, and $W_{B}(a)$ denotes the set of weak $\mathcal{B}$-inverses of $a$. Now $W_{B}(a) \neq \emptyset$ for any $a \in S(P)$.

Let $\rho$ be a strongly regular $\mathcal{P}$-congruence on $S(P)$. The set $\{p \rho: p \in P\}$ is denoted by $\mathcal{B}_{\rho}$. The following lemma also gives the examples of $\mathcal{P}$-kernel normal systems for $S(P)$. 
Lemma 2.1 If $\rho$ is a strongly regular $\mathcal{P}$-congruence on $S(P)$, then $\mathcal{B}_{\rho}$ is a $\mathcal{P}$-kernel normal system for $S(P)$.

Proof (K1) and (K2) are clear.

(K3) Since $\rho$ is a strongly regular $\mathcal{P}$-congruence, for any $a \in S(P)$ there exists $a^{\prime} \in W_{P}(a)$ such that $a \rho a a^{\prime} a$. Obviously, $a^{\prime} \in W_{B}(a)$.

(K4) Let $b=x b_{i_{1}} b_{i_{2}} \cdots b_{i_{n}} y \in x B_{i_{1}} B_{i_{2}} \cdots B_{i_{n}} y \cap B_{j}$, where $b_{i_{t}} \in B_{i_{t}}, t=$ $1,2, \ldots, n$, and let $B_{j}=p \rho, p \in P$. Then $b \rho p$. For any $c=x c_{i_{1}} c_{i_{2}} \cdots c_{i_{n}} y \in$ $x B_{i_{1}} B_{i_{2}} \cdots B_{i_{n}} y$, where $c_{i_{t}} \in B_{i_{t}}, t=1,2, \ldots, n$, we have that $b_{i_{t}}, c_{i_{t}} \in B_{i_{t}}$ implies $b_{i_{t}} \rho=c_{i_{t}} \rho$, and so that $c \rho=x \rho c_{i_{1}} \rho c_{i_{2}} \rho \cdots c_{i_{n}} \rho y \rho=x \rho b_{i_{1}} \rho b_{i_{2}} \rho \cdots b_{i_{n}} \rho y \rho=$ $b \rho=p \rho$. Hence $c \in B_{j}$.

We have the following results on $\mathcal{P}$-kernel normal systems which will be useful in the sequel.

Lemma 2.2 Let $\mathcal{B}=\left\{B_{i}: i \in I\right\}$ be a $\mathcal{P}$-kernel normal system for $S(P)$.

(1) If $a^{\prime} \in W_{B}(a), b^{\prime} \in W_{B}(b)$, then $a a^{\prime} a \in W_{B}\left(a^{\prime}\right), b^{\prime} a^{\prime} \in W_{B}(a b)$.

(2) Let $x, y \in S^{1}, i_{1}, i_{2}, \ldots, i_{n} \in I$. If $x B_{i_{1}} B_{i_{2}} \cdots B_{i_{n}} y \cap P \neq \emptyset$, then there exists $j \in I$ such that $x B_{i_{1}} B_{i_{2}} \cdots B_{i_{n}} y \subseteq B_{j}$.

(3) For $a \in S(P), a^{\prime} \in W_{B}(a)$ and $i \in I$, there exist $j, k \in I$ such that $a B_{i} a^{\prime} \subseteq B_{j}$, and $a^{\prime} B_{i} a \subseteq B_{k}$.

(4) If $a \in B_{i}, a^{\prime} \in W_{B}(a)$ then there exist $j, k \in I$ such that $a^{\prime} B_{i} \subseteq B_{j}$ and $B_{i} a^{\prime} \subseteq B_{k}$.

(5) If $a, a b \in B_{i}$ and $a b^{\prime}, b b^{\prime} \in B_{j}$ for some $b^{\prime} \in W_{B}(b), i, j \in I$, then $b \in B_{i}$.

(6) If $a, b a \in B_{i}$ and $b^{\prime} a, b^{\prime} b \in B_{j}$ for some $b^{\prime} \in W_{B}(b), i, j \in I$, then $b \in B_{i}$.

Proof (1) Let $a^{\prime} \in W_{B}(a), b^{\prime} \in W_{B}(b)$. It is easy to see that $a a^{\prime} a \in V\left(a^{\prime}\right)$ and $a a^{\prime} a \in$ $W_{P}\left(a^{\prime}\right)$. Thus $a a^{\prime} a \in W_{B}\left(a^{\prime}\right)$.

Now $a^{\prime} \in W_{P}(a), b^{\prime} \in W_{P}(b)$. It follows from [4, Lemma 1.4] that $b^{\prime} a^{\prime} \in W_{P}(a b)$. Let $x, y \in S^{1}$. Then

$x a b b^{\prime} a^{\prime} a b y \in B_{i} \Leftrightarrow x a a^{\prime} a b b^{\prime} a^{\prime} a b b^{\prime} b y \in B_{i} \quad\left(\right.$ since $a^{\prime} \in W_{B}(a)$ and $\left.b^{\prime} \in W_{B}(b)\right)$

$$
\begin{aligned}
& \Leftrightarrow x a\left(a^{\prime} a b b^{\prime}\right)^{2} b y \in B_{i} \\
& \Leftrightarrow x a a^{\prime} a b b^{\prime} b y \in B_{i} \quad\left(\text { since } a^{\prime} a b b^{\prime} \in P^{2} \subseteq E(S)\right) \\
& \Leftrightarrow x a b y \in B_{i} \quad\left(\text { since } a^{\prime} \in W_{B}(a) \text { and } b^{\prime} \in W_{B}(b)\right) .
\end{aligned}
$$

Hence $b^{\prime} a^{\prime} \in W_{B}(a b)$.

(2) By (K2), each element of $P$ is contained in some $B_{j}$. The result follows from (K4).

(3) Suppose that $B_{i}$ contains some element $p \in P$. Then $a p a^{\prime} \in P$. Hence $a B_{i} a^{\prime} \cap P \neq \emptyset$. Now it follows from (2) that there exists $j \in I$ such that $a B_{i} a^{\prime} \subseteq B_{j}$. Similarly, we can show the other result.

(4) Since $a \in B_{i}$ and $a^{\prime} \in W_{B}(a), a^{\prime} a \in a^{\prime} B_{i} \cap P$. It follows from (2) that there exists $B_{j} \in \mathcal{B}$ such that $a^{\prime} B_{i} \subseteq B_{j}$. Similarly, we can prove the other result. 
(5) Since $a b \in B_{i}, a b b^{\prime} b \in B_{i}$. Now $b^{\prime} b \in P$, by (K2), there exists $B_{k} \in \mathcal{B}$ such that $b^{\prime} b \in B_{k}$. Hence $a b^{\prime} b, a b b^{\prime} b \in B_{i} B_{k}$, and so $B_{i} B_{k} \cap B_{i} \neq \emptyset$. By (K4), $B_{i} B_{k} \subseteq$ $B_{i}$, so that $a b^{\prime} b \in B_{i}$. Now $a b^{\prime} b \in B_{j} b$. Thus $B_{j} b \cap B_{i} \neq \emptyset$. By (K4), $B_{j} b \subseteq B_{i}$. Since $b b^{\prime} \in B_{j}, b b^{\prime} b \in B_{i}$, that is, $b \in B_{i}$.

(6) Similar to the proof of (5), we can obtain (6).

We now introduce the following notation. Let $\mathcal{B}=\left\{B_{i}: i \in I\right\}$ be a $\mathcal{P}$-kernel normal system for $S(P)$ and $a, b \in S(P)$. By $a \sim b$ we mean that $a$ and $b$ are contained in a same $B_{i}$. Define a relation $\rho_{\mathcal{B}}$ on $S(P)$ by

$$
a \rho_{\mathcal{B}} b \Leftrightarrow\left(\exists a^{\prime} \in W_{B}(a)\right)\left(\exists b^{\prime} \in W_{B}(b)\right) b a^{\prime} \sim a a^{\prime} \& b^{\prime} a \sim b^{\prime} b .
$$

Clearly, $\rho_{\mathcal{B}}$ is a reflexive relation on $S(P)$.

In order to obtain the main result in this paper, we shall set up a series of lemmas.

\section{Lemma $2.3 \rho_{\mathcal{B}}$ is a symmetric relation on $S(P)$.}

Proof Let $a \rho_{\mathcal{B}} b$. Then there exist $a^{\prime} \in W_{B}(a), b^{\prime} \in W_{B}(b)$ such that $b a^{\prime} \sim a a^{\prime}$ and $b^{\prime} a \sim b^{\prime} b$. Then there exist $B_{i_{1}}, B_{i_{2}} \in \mathcal{B}$ such that $b a^{\prime}, a a^{\prime} \in B_{i_{1}}$ and $b^{\prime} a, b^{\prime} b \in B_{i_{2}}$. Let $a^{+} \in W_{B}(a)$ and $b^{+} \in W_{B}(b)$. Now by Lemma 2.2(1), $a a^{\prime} a b^{+} \in W_{B}\left(b a^{\prime}\right)$. Then by Lemma 2.2(4), $B_{i_{1}}\left(a a^{\prime} a b^{+}\right) \subseteq B_{i_{3}}$ for some $i_{3} \in I$. Now $a a^{\prime} a b^{+}=$ $\left(a a^{\prime}\right) a a^{\prime} a b^{+} \in B_{i_{1}}\left(a a^{\prime} a b^{+}\right) \subseteq B_{i_{3}}$. By $(\mathrm{K} 3), a b^{+} \in B_{i_{3}}$. Also, $b a^{\prime} a b^{+}=$ $\left(b a^{\prime}\right)\left(a a^{\prime} a\right) b^{+} \in B_{i_{3}}$. Then by $b^{\prime} \in W_{B}(b), b\left(b^{\prime} b\right)\left(a^{\prime} a\right) b^{+} \in B_{i_{3}}$. Hence $b B_{i_{2}} B_{i_{4}} b^{+} \cap$ $B_{i_{3}} \neq \emptyset$, where $a^{\prime} a \in B_{i_{4}}$. By (K4), $b B_{i_{2}} B_{i_{4}} b^{+} \subseteq B_{i_{3}}$. Hence $b b^{\prime} a a^{\prime} a b^{+} \in B_{i_{3}}$, that is, $b b^{\prime} a b^{+} \in B_{i_{3}}$. Hence $a b^{+}, b b^{\prime} a b^{+} \in B_{i_{3}}$. Now $b^{\prime} a \sim b^{\prime} b$ implies that $b\left(b^{\prime} b\right) b^{+} \sim b\left(b^{\prime} a\right) b^{+}$by Lemma 2.2(3). Therefore $a b^{+} \sim b b^{\prime} a b^{+} \sim b b^{\prime} b b^{+} \sim b b^{+}$, and so $a b^{+} \sim b b^{+}$. Similarly, we can prove that $a^{+} b \sim a^{+} a$. Hence $b \rho_{\mathcal{B}} a$, and so that $\rho_{\mathcal{B}}$ is symmetric.

Remark In the proof of Lemma 2.3, we also prove that if there exist $a^{\prime} \in W_{B}(a), b^{\prime} \in$ $W_{B}(b)$ such that $b a^{\prime} \sim a a^{\prime}$ and $b^{\prime} a \sim b^{\prime} b$, then $a b^{+} \sim b b^{+}$and $a^{+} b \sim a^{+} a$ for all $a^{+} \in W_{B}(a), b^{+} \in W_{B}(b)$. In the similar way, we can prove that if there exist $a^{+} \in W_{B}(a), b^{+} \in W_{B}(b)$ such that $a b^{+} \sim b b^{+}$and $a^{+} b \sim a^{+} a$, then $b a^{\prime} \sim a a^{\prime}$ and $b^{\prime} a \sim b^{\prime} b$ for all $a^{\prime} \in W_{B}(a), b^{\prime} \in W_{B}(b)$. In the previous definition of $\rho_{\mathcal{B}}$ we can substitute "there exists" by "for all".

Lemma 2.4 If $(a, b),(b, c) \in \rho_{\mathcal{B}}$, then $a\left(c^{\prime} c\right) a^{\prime} \sim a a^{\prime}, c\left(a^{\prime} a\right) c^{\prime} \sim c c^{\prime}, a^{\prime}\left(c c^{\prime}\right) a \sim$ $a^{\prime} a, b^{\prime}\left(a a^{\prime}\right) b \sim b^{\prime} b$ for all $a^{\prime} \in W_{B}(a), b^{\prime} \in W_{B}(b), c^{\prime} \in W_{B}(c)$.

Proof Since $(a, b),(b, c) \in \rho_{\mathcal{B}}, b a^{\prime} \sim a a^{\prime}, b^{\prime} a \sim b^{\prime} b, a^{\prime} b \sim a^{\prime} a, a b^{\prime} \sim b b^{\prime}, c b^{\prime} \sim$ $b b^{\prime}, c^{\prime} b \sim c^{\prime} c, b^{\prime} c \sim b^{\prime} b, b c^{\prime} \sim c c^{\prime}$ for all $a^{\prime} \in W_{B}(a), b^{\prime} \in W_{B}(b)$ and $c^{\prime} \in W_{B}(c)$ (from the remark after Lemma 2.3). So there exist $B_{i_{1}}, B_{i_{2}}, B_{i_{3}}, B_{i_{4}}, B_{i_{5}}, B_{i_{6}}, B_{i_{7}}$ and $B_{i_{8}}$ in $\mathcal{B}$ such that $b a^{\prime}, a a^{\prime} \in B_{i_{1}}, b^{\prime} a, b^{\prime} b \in B_{i_{2}}, a^{\prime} b, a^{\prime} a \in B_{i_{3}}, a b^{\prime}, b b^{\prime} \in$ $B_{i_{4}}, c b^{\prime}, b b^{\prime} \in B_{i_{5}}, c^{\prime} b, c^{\prime} c \in B_{i_{6}}, b^{\prime} c, b^{\prime} b \in B_{i_{7}}$ and $b c^{\prime}, c c^{\prime} \in B_{i_{8}}$. From (K1), $B_{i_{2}}=B_{i_{7}}$ and $B_{i_{4}}=B_{i_{5}}$. 
We first show that $a\left(c^{\prime} c\right) a^{\prime} \sim a a^{\prime}$. Now $a\left(a^{\prime} b\right)\left(b^{\prime} b\right)\left(c^{\prime} c\right) a^{\prime}, a\left(a^{\prime} b\right)\left(b^{\prime} c\right)\left(c^{\prime} c\right) a^{\prime}$, $a\left(a^{\prime} a\right)\left(b^{\prime} c\right)\left(c^{\prime} b\right) a^{\prime} \in a B_{i_{3}} B_{i_{2}} B_{i_{6}} a^{\prime}$. Since $a\left(b^{\prime} c\right)\left(c^{\prime} b\right) a^{\prime} \in P$, by (K2), there exists $i_{9} \in I$ such that $a\left(b^{\prime} c\right)\left(c^{\prime} b\right) a^{\prime} \in B_{i_{9}}$, and so $a\left(a^{\prime} a\right)\left(b^{\prime} c\right)\left(c^{\prime} b\right) a^{\prime} \in B_{i_{9}}$ by (K3). Thus $a B_{i_{3}} B_{i_{2}} B_{i_{6}} a^{\prime} \cap B_{i_{9}} \neq \emptyset$. Hence from (K4), $a B_{i_{3}} B_{i_{2}} B_{i_{6}} a^{\prime} \subseteq B_{i_{9}}$, so that $a\left(a^{\prime} b\right)\left(b^{\prime} b\right)\left(c^{\prime} c\right) a^{\prime}, a\left(a^{\prime} b\right)\left(b^{\prime} c\right)\left(c^{\prime} c\right) a^{\prime} \in B_{i 9}$, that is, $a\left(a^{\prime} b\right)\left(c^{\prime} c\right) a^{\prime}, a\left(a^{\prime} b\right)\left(b^{\prime} c\right) a^{\prime} \in$ $B_{i_{9}}$.

Now $a\left(a^{\prime} a\right)\left(c^{\prime} c\right) a^{\prime}, a\left(a^{\prime} b\right)\left(c^{\prime} c\right) a^{\prime} \in a B_{i_{3}} B_{i_{6}} a^{\prime}$. Since $a\left(c^{\prime} c\right) a^{\prime} \in P$, there exists $i_{10} \in I$ such that $a\left(c^{\prime} c\right) a^{\prime} \in B_{i_{10}}$. Then by (K3), $a\left(a^{\prime} a\right)\left(c^{\prime} c\right) a^{\prime} \in B_{i_{10}}$. Hence $a B_{i_{3}} B_{i_{6}} a^{\prime} \cap B_{i_{10}} \neq \emptyset$. Then by (K4), $a B_{i_{3}} B_{i_{6}} a^{\prime} \subseteq B_{i_{10}}$. Thus $a\left(a^{\prime} b\right)\left(c^{\prime} c\right) a^{\prime} \in B_{i_{10}}$. By (K1), $B_{i_{10}}=B_{i_{9}}$. Hence $a\left(a^{\prime} a\right)\left(c^{\prime} c\right) a^{\prime} \in B_{i_{9}}$, and so $a\left(c^{\prime} c\right) a^{\prime} \in B_{i_{9}}$.

Now $a\left(a^{\prime} b\right)\left(b^{\prime} b\right) a^{\prime}, a\left(a^{\prime} b\right)\left(b^{\prime} c\right) a^{\prime}, a\left(a^{\prime} a\right)\left(b^{\prime} b\right) a^{\prime} \in a B_{i_{3}} B_{i_{7}} a^{\prime}$. Note $a\left(b^{\prime} b\right) a^{\prime} \in P$, there exists $i_{11} \in I$ such that $a\left(b^{\prime} b\right) a^{\prime} \in B_{i_{11}}$. By (K3), $a\left(a^{\prime} a\right)\left(b^{\prime} b\right) a^{\prime} \in B_{i_{11}}$, and so $a B_{i_{3}} B_{i_{7}} a^{\prime} \cap B_{i_{11}} \neq \emptyset$. Then by (K4), $a B_{i_{3}} B_{i_{7}} a^{\prime} \subseteq B_{i_{11}}$. Hence $a\left(a^{\prime} b\right)\left(b^{\prime} c\right) a^{\prime} \in$ $B_{i_{11}}$. By (K1), $B_{i_{11}}=B_{i_{9}}$. Then $a\left(c^{\prime} c\right) a^{\prime} \sim a\left(a^{\prime} b\right)\left(b^{\prime} b\right) a^{\prime} \sim a\left(a^{\prime} b\right) a^{\prime}$. Now $a^{\prime} b \sim a^{\prime} a$ implies (by Lemma 2.2(3)) that $a\left(a^{\prime} b\right) a^{\prime} \sim a\left(a^{\prime} a\right) a^{\prime}=a a^{\prime}$. Hence $a\left(c^{\prime} c\right) a^{\prime} \sim a a^{\prime}$.

Similarly, we can prove the other results.

Lemma $2.5 \rho_{\mathcal{B}}$ is a transitive relation on $S(P)$.

Proof Here we use all the notation of Lemma 2.4. Let $(a, b) \in \rho_{\mathcal{B}}$ and $(b, c) \in \rho_{\mathcal{B}}$. Let $x=b c^{\prime} c b^{\prime} a a^{\prime}$. Now $\left(c b^{\prime}\right)\left(a a^{\prime}\right),\left(b b^{\prime}\right)\left(b a^{\prime}\right) \in B_{i_{5}} B_{i_{1}}$ and $\left(b b^{\prime}\right)\left(b a^{\prime}\right) \sim b a^{\prime} \in B_{i_{1}}$. Hence $B_{i_{5}} B_{i_{1}} \cap B_{i_{1}} \neq \emptyset$. Then by (K4), $B_{i_{5}} B_{i_{1}} \subseteq B_{i_{1}}$. Hence $\left(c b^{\prime}\right)\left(a a^{\prime}\right) \in B_{i_{1}}$. This implies that $x=\left(b c^{\prime}\right)\left(c b^{\prime} a a^{\prime}\right),\left(c c^{\prime}\right)\left(c b^{\prime} a a^{\prime}\right) \in B_{i_{8}} B_{i_{1}}$. But $c b^{\prime} a a^{\prime} \sim\left(c c^{\prime}\right)\left(c b^{\prime} a a^{\prime}\right)$. Hence $B_{i_{8}} B_{i_{1}} \cap B_{i_{1}} \neq \emptyset$. Then by (K4), $B_{i_{8}} B_{i_{1}} \subseteq B_{i_{1}}$. Hence $x \in B_{i_{1}}$.

Let $y=c a^{\prime}$. By Lemma 2.2(1), $a a^{\prime} a c^{\prime} \in W_{B}(y)$. Let $y^{\prime}=a a^{\prime} a c^{\prime}$. Now $a^{\prime} b \sim a^{\prime} a$ implies (by Lemma 2.2(3)) $c\left(a^{\prime} b\right) c^{\prime} \sim c\left(a^{\prime} a\right) c^{\prime}$. From Lemma 2.4, $c a^{\prime} a c^{\prime} \sim c c^{\prime}$. Hence $c\left(a^{\prime} b\right) c^{\prime}, c c^{\prime} \in B_{i_{8}}$. Now $\left(c b^{\prime}\right)\left(a a^{\prime}\right),\left(b b^{\prime}\right)\left(b a^{\prime}\right) \in B_{i_{5}} B_{i_{1}}$. But $\left(b b^{\prime}\right)\left(b a^{\prime}\right) \sim$ $b a^{\prime} \in B_{i_{1}}$. Hence $B_{i_{5}} B_{i_{1}} \cap B_{i_{1}} \neq \emptyset$, and so $\left(c b^{\prime}\right)\left(a a^{\prime}\right) \in B_{i_{1}}$. Then $\left(c a^{\prime} b c^{\prime}\right)\left(c b^{\prime}\right)\left(a a^{\prime}\right)$, $\left(c c^{\prime}\right)\left(c b^{\prime}\right)\left(a a^{\prime}\right) \in B_{i_{8}} B_{i_{1}}$. But $\left(c c^{\prime}\right)\left(c b^{\prime}\right)\left(a a^{\prime}\right) \sim\left(c b^{\prime}\right)\left(a a^{\prime}\right) \in B_{i_{1}}$. Thus $B_{i_{8}} B_{i_{1}} \cap$ $B_{i_{1}} \neq \emptyset$, so that $B_{i_{8}} B_{i_{1}} \subseteq B_{i_{1}}$. Hence

$$
y x=c a^{\prime} b c^{\prime} c b^{\prime} a a^{\prime} \in B_{i_{1}} .
$$

Now $x, y x \in B_{i_{1}}$ implies that

$$
y x \sim x .
$$

To show that $y^{\prime} y \sim y^{\prime} x$, we notice that

$$
a\left(c^{\prime} b\right)\left(c^{\prime} c\right)\left(b^{\prime} c\right) a^{\prime}, a\left(c^{\prime} b\right)\left(c^{\prime} c\right)\left(b^{\prime} a\right) a^{\prime}, a\left(c^{\prime} c\right)\left(c^{\prime} b\right)\left(b^{\prime} b\right) a^{\prime} \in a B_{i_{6}} B_{i_{6}} B_{i 7} a^{\prime} .
$$

Here $a\left(c^{\prime} b\right)\left(c^{\prime} c\right)\left(b^{\prime} c\right) a^{\prime}=a\left(c^{\prime}\left(b\left(c^{\prime} c\right) b^{\prime}\right) c\right) a^{\prime} \in P$. Hence by Lemma 2.2(2), there exists $i_{12}$ such that $a B_{i_{6}} B_{i_{6}} B_{i_{7}} a^{\prime} \subseteq B_{i_{12}}$. Hence $a\left(c^{\prime} c\right)\left(c^{\prime} b\right)\left(b^{\prime} b\right) a^{\prime} \in B_{i_{12}}$, that is, $a c^{\prime} b a^{\prime} \in B_{i_{12}}$.

Now $c^{\prime} c \sim c^{\prime} b$. Hence by Lemma 2.2(3), $a\left(c^{\prime} c\right) a^{\prime} \sim a\left(c^{\prime} b\right) a^{\prime}$. But $a\left(c^{\prime} c\right) a^{\prime}, a a^{\prime} \in$ $B_{i_{1}}$ by Lemma 2.4. Hence $a\left(c^{\prime} b\right) a^{\prime} \in B_{i_{1}}$. By (K1), $B_{i_{1}}=B_{i_{12}}$. Consequently, $a c^{\prime} b c^{\prime} c b^{\prime} a a^{\prime} \in B_{i_{1}}$, and so, $a a^{\prime} a c^{\prime} b c^{\prime} c b^{\prime} a a^{\prime} \in B_{i_{1}}$. Hence $y^{\prime} x, x \in B_{i_{1}}$. Again 
$a c^{\prime} c a^{\prime} \sim a a^{\prime}$. Then $y^{\prime} y=a a^{\prime} a c^{\prime} c a^{\prime} \sim a c^{\prime} c a^{\prime} \sim a a^{\prime} \sim x$. Therefore

$$
y^{\prime} x \sim y^{\prime} y .
$$

From (2.1), (2.2), by Lemma 2.2(6), we have $y \sim x$. Then

$$
c a^{\prime} \sim x \sim a a^{\prime} .
$$

Next we show that $c^{\prime} a \sim c^{\prime} c$. Let $x_{1}=c^{\prime} c b^{\prime} a a^{\prime} b, y_{1}=c^{\prime} a$. Now $a^{\prime} c c^{\prime} c \in W_{B}\left(y_{1}\right)$. Let $y_{1}^{\prime}=a^{\prime} c c^{\prime} c$. From Lemma 2.4, $b^{\prime} a a^{\prime} b \sim b^{\prime} b \in B_{i 7}$. Hence $\left(c^{\prime} c\right)\left(b^{\prime} a a^{\prime} b\right)$, $\left(c^{\prime} c\right)\left(b^{\prime} a\right),\left(c^{\prime} b\right)\left(b^{\prime} b\right) \in B_{i_{6}} B_{i_{7}}$. But $\left(c^{\prime} b\right)\left(b^{\prime} b\right) \sim c^{\prime} b \in B_{i_{6}}$, and so $B_{i_{6}} B_{i_{7}} \cap B_{i_{6}} \neq \emptyset$. Then (K4) implies that $B_{i_{6}} B_{i_{7}} \subseteq B_{i_{6}}$, so that $x_{1}=\left(c c^{\prime}\right)\left(b^{\prime} a a^{\prime} b\right) \in B_{i_{6}}$.

Now $x_{1} y_{1}=\left(c^{\prime} c b^{\prime} a\right)\left(a^{\prime} b\right)\left(c^{\prime} a\right)=\left(c^{\prime} c\right)\left(b^{\prime} a\right)\left(a^{\prime} b c^{\prime} a\right)$. Here $a^{\prime}\left(b c^{\prime}\right) a \sim a^{\prime}\left(c c^{\prime}\right) a$ (by Lemma 2.2(3)) $\sim a^{\prime} a$ (by Lemma 2.4) $\in B_{i_{3}}$. Notice that $\left(c^{\prime} c\right)\left(b^{\prime} a\right) \in B_{i_{6}}$ and so $x_{1} y_{1} \in B_{i_{6}} B_{i_{3}}$. Also, $\left(c^{\prime} c\right)\left(b^{\prime} a\right)\left(a^{\prime} a\right) \in B_{i_{6}} B_{i_{3}}$, and $\left(c^{\prime} c\right)\left(b^{\prime} a\right)\left(a^{\prime} a\right) \sim\left(c^{\prime} c\right)\left(b^{\prime} a\right) \in$ $B_{i_{6}}$. Hence $B_{i_{6}} B_{i_{3}} \subseteq B_{i_{6}}$, so that $x_{1} y_{1} \in B_{i_{6}}$. Now $x_{1}, x_{1} y_{1} \in B_{i_{6}}$ implies that

$$
x_{1} y_{1} \sim x_{1} \text {. }
$$

To show that $x_{1} y_{1}^{\prime} \sim y_{1} y_{1}^{\prime}$, notice that $x_{1} y_{1}^{\prime}=c^{\prime} c b^{\prime} a a^{\prime} b a^{\prime} c c^{\prime} c$. Now $\left(c b^{\prime}\right)\left(a a^{\prime}\right)$, $\left(b b^{\prime}\right)\left(b a^{\prime}\right) \in B_{i_{5}} B_{i_{1}}$. But $\left(b b^{\prime}\right)\left(b a^{\prime}\right) \sim b a^{\prime} \in B_{i_{1}}$. Hence $\left(c b^{\prime}\right)\left(a a^{\prime}\right), b a^{\prime} \in B_{i_{1}}$. So $c b^{\prime} a a^{\prime} b a^{\prime}=\left(c b^{\prime} a a^{\prime}\right)\left(b a^{\prime}\right) \in B_{i_{1}} B_{i_{1}} \subseteq B_{i_{1}}$ (since $B_{i_{1}}$ is a subsemigroup). Then $c b^{\prime} a a^{\prime} b a^{\prime} \sim a a^{\prime}$. It follows from Lemma 2.2(3) that $c^{\prime}\left(c b^{\prime} a a^{\prime} b a^{\prime}\right) c \sim c^{\prime}\left(a a^{\prime}\right) c$. Hence $c^{\prime}\left(c b^{\prime} a a^{\prime} b a^{\prime}\right) c c^{\prime} c \sim c^{\prime}\left(a a^{\prime}\right) c c^{\prime} c$, that is,

$$
x_{1} y_{1}^{\prime} \sim y_{1} y_{1}^{\prime} .
$$

From (2.3), (2.4), by Lemma 2.2(5), we have that $x_{1} \sim y_{1}$, and so $c^{\prime} c \sim x_{1} \sim c^{\prime} a$. Hence $(a, c) \in \rho_{\mathcal{B}}$.

Lemma 2.6 $\rho_{\mathcal{B}}$ is a strongly regular $\mathcal{P}$-congruence on $S(P)$.

Proof It follows from Lemma 2.3 and Lemma 2.5 that $\rho_{\mathcal{B}}$ is an equivalence relation on $S(P)$. Let us show that $\rho_{\mathcal{B}}$ is left compatible. Let $(a, b) \in \rho_{\mathcal{B}}, c \in S$, $a^{\prime} \in W_{B}(a), b^{\prime} \in W_{B}(b)$ and $c^{\prime} \in W_{B}(c)$. Then $b a^{\prime} \sim a a^{\prime}, b^{\prime} a \sim b^{\prime} b, a^{\prime} b \sim a^{\prime} a$, $a b^{\prime} \sim b b^{\prime}$. So there exist $B_{i_{1}}, B_{i_{2}}, B_{i_{3}}, B_{i_{4}} \in \mathcal{B}$ such that $b a^{\prime}, a a^{\prime} \in B_{i_{1}}, b^{\prime} a, b^{\prime} b \in$ $B_{i_{2}}, a^{\prime} b, a^{\prime} a \in B_{i_{3}}, a b^{\prime}, b b^{\prime} \in B_{i_{4}}$. Now $b a^{\prime} \sim a a^{\prime}$ implies that $c\left(b a^{\prime}\right) c^{\prime} \sim c\left(a a^{\prime}\right) c^{\prime}$ (by Lemma 2.2(3)).

We show that $b^{\prime} c^{\prime} c a \sim b^{\prime} c^{\prime} c b$ as follows. Let $x=b^{\prime} c^{\prime} c b a^{\prime} a, y=b^{\prime} c^{\prime} c a, y^{\prime}=$ $a^{\prime} c^{\prime} c b b^{\prime} b$. Then $y^{\prime} \in W_{B}(y)$. Notice that $b^{\prime} c^{\prime} c b \in P$. Let $b^{\prime} c^{\prime} c b \in B_{i_{5}} \in \mathcal{B}$. Now $\left(b^{\prime} c^{\prime} c b\right)\left(b^{\prime} a\right),\left(b^{\prime} c^{\prime} c b\right)\left(b^{\prime} b\right) \in B_{i_{5}} B_{i_{2}}$. Since $\left(b^{\prime} c^{\prime} c b\right)\left(b^{\prime} b\right) \sim b^{\prime} c^{\prime} c b \in B_{i_{5}}, B_{i_{5}} B_{i_{2}} \cap$ $B_{i_{5}} \neq \varnothing$. Hence by $(\mathrm{K} 4), \quad B_{i_{5}} B_{i_{2}} \subseteq B_{i_{5}}$. Then $\left(b^{\prime} c^{\prime} c b\right)\left(b^{\prime} a\right) \in B_{i_{5}}$. Now $\left(b^{\prime} c^{\prime} c b\right)\left(b^{\prime} b\right)\left(a^{\prime} a\right),\left(b^{\prime} c^{\prime} c b\right)\left(b^{\prime} a\right)\left(a^{\prime} a\right) \in B_{i_{5}} B_{i_{3}}$. But $\left(b^{\prime} c^{\prime} c b\right)\left(b^{\prime} a\right)\left(a^{\prime} a\right) \sim$ $\left(b^{\prime} c^{\prime} c b\right)\left(b^{\prime} a\right) \in B_{i_{5}}$ shows that $B_{i_{5}} B_{i_{3}} \cap B_{i_{5}} \neq \emptyset$. Then $B_{i_{5}} B_{i_{3}} \subseteq B_{i_{5}}$ and $\left(b^{\prime} c^{\prime} c b\right)\left(b^{\prime} b\right)\left(a^{\prime} a\right) \in B_{i_{5}}$, that is, $x=b^{\prime} c^{\prime} c b a^{\prime} a \in B_{i_{5}}$.

Now $a b^{\prime} \sim b b^{\prime}$, and $b^{\prime} c^{\prime} c \in W_{B}\left(c^{\prime} c b\right)$. Hence by Lemma 2.2(3), $b^{\prime}\left(c^{\prime} c\left(a b^{\prime}\right) c^{\prime} c\right) b$ $\sim b^{\prime}\left(c^{\prime} c\left(b b^{\prime}\right) c^{\prime} c\right) b$. Since $b^{\prime} c^{\prime} c b \in P \subseteq E_{S}, b^{\prime}\left(c^{\prime} c\left(b b^{\prime}\right) c^{\prime} c\right) b=\left(b^{\prime} c^{\prime} c b\right)^{2}=b^{\prime} c^{\prime} c b$. 
Now $b^{\prime} c^{\prime} c b \in B_{i_{5}}$, hence $b^{\prime}\left(c^{\prime} c\left(a b^{\prime}\right) c^{\prime} c\right) b \in B_{i_{5}}$. Then $y x=\left(b^{\prime} c^{\prime} c a\right)\left(b^{\prime} c^{\prime} c b a^{\prime} a\right)=$ $\left(b^{\prime}\left(c^{\prime} c a b^{\prime} c^{\prime} c\right) b\right)\left(a^{\prime} a\right) \in B_{i_{5}} B_{i_{3}}$. But $B_{i_{5}} B_{i_{3}} \subseteq B_{i_{5}}$ and so $y x \in B_{i_{5}}$. Then

$$
y x \sim x \sim b^{\prime} c^{\prime} c b
$$

To show that $y^{\prime} x \sim y^{\prime} y$, notice that $\left(c^{\prime} c\right)\left(b b^{\prime}\right)\left(c^{\prime} c\right) \in P$. Then there exists $B_{i_{6}} \in \mathcal{B}$ such that $\left(c^{\prime} c\right)\left(b b^{\prime}\right)\left(c^{\prime} c\right) \in B_{i_{6}}$. Now $y^{\prime} x=a^{\prime} c^{\prime} c b b^{\prime} b b^{\prime} c^{\prime} c b a^{\prime} a=a^{\prime}\left(c^{\prime} c b b^{\prime} c^{\prime} c\right)\left(b a^{\prime}\right) a$, $a^{\prime}\left(c^{\prime} c b b^{\prime} c^{\prime} c\right)\left(a a^{\prime}\right) a \in a^{\prime} B_{i_{6}} B_{i_{1}} a$. Notice that $y^{\prime} y=a^{\prime} c^{\prime} c b b^{\prime} b b^{\prime} c^{\prime} c a=$ $a^{\prime}\left(c^{\prime} c b b^{\prime} c^{\prime} c\right) a \in P$. Then there exists $B_{i_{7}} \in \mathcal{B}$ such that $y^{\prime} y \in B_{i_{7}}$, and so $a^{\prime}\left(c^{\prime} c b b^{\prime} c^{\prime} c\right)\left(a a^{\prime}\right) a \in B_{i_{7}}$. Hence $a^{\prime} B_{i_{6}} B_{i_{1}} a \subseteq B_{i_{7}}$. Then

$$
y^{\prime} x \sim y^{\prime} y \text {. }
$$

From (2.5), (2.6), by Lemma 2.2(6), $y \sim x \sim b^{\prime} c^{\prime} c b$, that is, $b^{\prime} c^{\prime} c a \sim b^{\prime} c^{\prime} c b$.

By Lemma 2.2(1), $a^{\prime} c^{\prime} \in W_{B}(c a), b^{\prime} c^{\prime} \in W_{B}(c b)$. Hence $(c a, c b) \in \rho_{\mathcal{B}}$, and so $\rho_{\mathcal{B}}$ is left compatible. A similar argument shows that $\rho_{\mathcal{B}}$ is right compatible.

It is easy to verify that $a^{\prime} \in W_{B}\left(a a^{\prime} a\right)$ and $\left(a, a a^{\prime} a\right) \in \rho_{\mathcal{B}}$. Hence $\rho_{\mathcal{B}}$ is a strongly regular $\mathcal{P}$-congruence on $S(P)$.

Lemma 2.7 If $p \in B_{i} \cap P$, then $p \rho_{\mathcal{B}}=B_{i}$.

Proof Let $a \in p \rho_{\mathcal{B}}$. Then $p \rho_{\mathcal{B}} a$. Since $p \in W_{B}(p)$, ap $\sim p p=p$ and $a^{\prime} p \sim a^{\prime} a$ for any $a^{\prime} \in W_{B}(a)$. Hence by Lemma 2.2(6), $a \in B_{i}$.

Conversely, let $b \in B_{i}$. Since $B_{i}$ is a subsemigroup, $p b \in B_{i}$. Then $p b \sim p p$. Let $b^{\prime} \in W_{B}(b)$. By Lemma 2.2(4), there exists $B_{j} \in \mathcal{B}$ such that $B_{i} b^{\prime} \subseteq B_{j}$. Hence $p b^{\prime}, b b^{\prime} \in B_{j}$, and so $p b^{\prime} \sim b b^{\prime}$. By the definition of $\rho_{\mathcal{B}},(b, p) \in \rho_{\mathcal{B}}$. Thus $b \in p \rho \mathcal{B}$.

Lemma 2.8 Let $\rho$ and $\sigma$ be two strongly regular $\mathcal{P}$-congruences on $S(P)$. Then $\rho=\sigma$ if and only if $\mathcal{B}_{\rho}=\mathcal{B}_{\sigma}$.

Proof It suffices to show the "if" part. Let $a \rho b$. Since $\sigma$ is a strongly regular $\mathcal{P}$ congruence, there exists $a^{\prime} \in W_{P}(a)$ such that $a \sigma a a^{\prime} a$. Now $a a^{\prime} \rho b a^{\prime}$. Note $a a^{\prime} \in P$. By the assumption, there exists $p \in P$ such that $a a^{\prime} \rho=p \sigma$. Hence $a a^{\prime} \rho=a a^{\prime} \sigma$, and so $b a^{\prime} \in a a^{\prime} \sigma$, that is, $a a^{\prime} \sigma b a^{\prime}$. Similarly, we have that $b \sigma b b^{\prime} b$ and $b^{\prime} a \sigma b^{\prime} b$ for some $b^{\prime} \in W_{P}(b)$. Thus

$$
a \sigma a a^{\prime} a \sigma b a^{\prime} a \sigma b b^{\prime} b a^{\prime} a \sigma b b^{\prime} a a^{\prime} a \sigma b b^{\prime} a \sigma b b^{\prime} b \sigma b .
$$

Hence $a \sigma b$, and so $\rho \subseteq \sigma$. Similarly, we have $\sigma \subseteq \rho$. Thus $\rho=\sigma$.

The following theorem is the main result in this paper.

Theorem 2.9 If $\mathcal{B}$ is a $\mathcal{P}$-kernel normal system for $S(P)$, then $\rho_{\mathcal{B}}$ is a strongly regular $\mathcal{P}$-congruence on $S(P)$ and $\mathcal{B}_{\rho_{\mathcal{B}}}=\mathcal{B}$. Conversely, if $\rho$ is a strongly regular $\mathcal{P}$-congruence on $S(P)$, then $\mathcal{B}_{\rho}$ is a $\mathcal{P}$-kernel normal system for $S(P)$ and $\rho_{\mathcal{B}_{\rho}}=\rho$. 
Proof Direct part. Let $\mathcal{B}=\left\{B_{i}: i \in I\right\}$ be a $\mathcal{P}$-kernel normal system for $S(P)$. By Lemma $2.6, \rho_{\mathcal{B}}$ is a strongly regular $\mathcal{P}$-congruence on $S(P)$.

Let $p \in P$. Then there exists $B_{i} \in \mathcal{B}$ such that $p \in B_{i}$. Hence by Lemma 2.7, $p \rho_{\mathcal{B}}=B_{i}$, and so $\mathcal{B}_{\rho_{\mathcal{B}}} \subseteq \mathcal{B}$. Conversely, suppose that $B_{i} \in \mathcal{B}$. Then $B_{i}$ contains some element $p \in P$. By Lemma 2.7 again, $B_{i}=p \rho_{\mathcal{B}}$, so that $\mathcal{B}_{\rho_{\mathcal{B}}}=\mathcal{B}$ as required.

Converse part. Let $\rho$ be a strongly regular $\mathcal{P}$-congruence on $S(P)$. Then it follows from Lemma 2.1 that $\mathcal{B}_{\rho}$ is a $\mathcal{P}$-kernel normal system for $S(P)$. From the direct part of this theorem, we have that $\rho_{\mathcal{B}}$ is also a strongly regular $\mathcal{P}$-congruence on $S(P)$ and $\mathcal{B}_{\rho_{\mathcal{B}}}=\mathcal{B}_{\rho}$. Hence by Lemma $2.8, \rho_{\mathcal{B}_{\rho}}=\rho$.

The set of all strongly regular $\mathcal{P}$-congruences on $S(P)$ and the set of all $\mathcal{P}$-kernel normal systems for $S(P)$ are denoted by $S R C_{P}(S)$ and $K N S_{P}(S)$, respectively.

Define a relation $\leq$ on $K N S_{P}(S)$ by

$$
\mathcal{A} \leq \mathcal{B} \Leftrightarrow(\forall A \in \mathcal{A})(\exists B \in \mathcal{B}) A \subseteq B
$$

It is clear that $\leq$ is a partial order on $K N S_{P}(S)$.

Now the next result follows immediately.

Corollary 2.10 The mappings

$$
\begin{aligned}
\varphi: \quad S R C_{P}(S) & \rightarrow K N S_{P}(S) \\
\rho & \mapsto \mathcal{B}_{\rho}
\end{aligned}
$$

and

$$
\begin{aligned}
\psi: \quad K N S_{P}(S) & \rightarrow S R C_{P}(S) \\
\mathcal{B} & \mapsto \rho_{\mathcal{B}}
\end{aligned}
$$

are mutually inverse order preserving bijections.

Acknowledgements The author would like to thank Professor Thomas E. Hall and the referee for their valuable suggestions and comments.

Open Access This article is distributed under the terms of the Creative Commons Attribution Noncommercial License which permits any noncommercial use, distribution, and reproduction in any medium, provided the original author(s) and source are credited.

\section{References}

1. Blyth, T.S., Almeida Santos, M.H.: E-inversive Dubreil-Jacotin semigroups. Semigroup Forum 78, 511-527 (2009)

2. Catino, F., Miccoli, M.M.: On semidirect products of semigroups. Note Math. 9, 189-194 (1989)

3. Fan, X.K., Chen, Q.H.: Regular $\mathcal{P}$-congruences on $\mathcal{P}$-inversive semigroups. Pure Appl. Math. 19, 374-378 (2003)

4. Fan, X.K., Chen, Q.H.: Strong $\mathcal{P}$-congruences on $\mathcal{P}$-inversive semigroups. Adv. Math. 33, 434-440 (2004)

5. Fan, X.K., Chen, Q.H.: $\mathcal{P}$-kernel normal system of strong $\mathcal{P}$-congruences on $\mathcal{P}$-inversive semigroups. Northeast. Math. J. 21, 397-403 (2005) 
6. Feigenbaum, R.: Regular semigroup congruences. Semigroup Forum 17, 373-377 (1979)

7. Gao, Z.H., Yu, B.J.: Sublattices of the lattices of strong $\mathcal{P}$-congruences on $\mathcal{P}$-inversive semigroups. Semigroup Forum 75, 272-292 (2007)

8. Hall, T.E., Munn, W.D.: The hypercore of a semigroup. Proc. Edinb. Math. Soc. 28, 107-122 (1985)

9. Hayes, A.D.: E*-dense E-semigroups. Semigroup Forum 68, 218-232 (2004)

10. Hayes, A.D.: Semigroups which have a minimum primitive inverse congruence. Semigroup Forum 65, 386-404 (2002)

11. Howie, J.M.: Fundamentals of Semigroup Theory. Clarendon, Oxford (1995)

12. Imaoka, T.: *-congruences on regular *-semigroups. Semigroup Forum 23, 321-326 (1981)

13. Luo, Y.F., Fan, X.K., Li, X.L.: Regular congruences on an E-inversive semigroup. Semigroup Forum 76, 107-123 (2008)

14. Meakin, J.: Congruences on orthodox semigroups. J. Aust. Math. Soc. 12, 323-341 (1971)

15. Mitsch, H.: Subdirect products of E-inversive semigroups. J. Aust. Math. Soc. 48, 66-78 (1990)

16. Mitsch, H.: On the lemma of Lallement. Commun. Algebra 24, 3085-3098 (1996)

17. Mitsch, H.: Introduction to E-inversive semigroups. In: Smith, P., Giraldes, E., Martins, P. (eds.) Proc. Conf. Semigroups. World Scientific, Singapore (2000)

18. Mitsch, H., Petrich, M.: Basic properties of E-inversive semigroups. Commun. Algebra 28, 5169$5182(2000)$

19. Nordahl, T.E., Scheiblich, H.E.: Regular *-semigroups. Semigroup Forum 28, 369-377 (1978)

20. Pastijn, F., Perich, M.: Congruences on regular semigroups. Trans. Am. Math. Soc. 295, 607-633 (1986)

21. Preston, G.B.: Inverse semigroups. J. Lond. Math. Soc. 29, 396-403 (1954)

22. Reither, S.: Die natürliche ordnung auf halbgruppen. Ph.D. Thesis, University of Vienna (1994)

23. Sen, M.K.: $\mathcal{P}$-congruences on $\mathcal{P}$-regular semigroups. Semigroup Forum 44, 149-156 (1992)

24. Siripitukdet, M., Sattayaporn, S.: The maximum idempotent-separating congruence on E-inversive E-semigroups. Int. J. Algebra 2, 925-931 (2008)

25. Thierrin, G.: Surles demi-groups inverses. C. R. Acad. Sci. Paris 234, 1336-1338 (1952)

26. Trotter, P.G.: Normal partitions of idempotents of regular semigroups. J. Aust. Math. Soc. A 26, 110114 (1978)

27. Weipoltshammer, B.: Certain congruences on E-inversive E-semigroups. Semigroup Forum 65, 233248 (2002)

28. Yamada, M., Sen, M.K.: $\mathcal{P}$-regular semigroups. Semigroup Forum 39, 157-178 (1989)

29. Zheng, H.W.: Strong $\mathcal{P}$-congruences on $\mathcal{P}$-regular semigroups. Semigroup Forum 51, 217-223 (1995)

30. Zheng, H.W.: Group congruences on an E-inversive semigroup. Southeast Asian Bull. Math. 21, 1-8 (1997) 